\title{
THE INFRARED EMISSION FEATURES AND POLYCYCLIC AROMATIC HYDROCARBONS
}

\author{
L. J. Allamandola \\ NASA-Ames Research Center, \\ Moffett Field, CA 94095
}

\begin{abstract}
The infrared evidence which supports the PAH hypothesis is briefly summarized. Rather than presenting a general discussion of these assignments, this paper focuses on the spectroscopic issues raised by recent observational and experimental developments. These issues include: the position and profile of the " $1310 " \mathrm{~cm}^{-1}(" 7.7 " \mu \mathrm{m})$ feature, the position and intensities of the bands in the $910-710 \mathrm{~cm}^{-1}(11-14 \mu \mathrm{m})$ region, the newly detected $1900 \mathrm{~cm}^{-1}(5.3 \mu \mathrm{m}) \mathrm{band}$, and the spatial and spectral variations in the $3000 \mathrm{~cm}^{-1}(3 \mu \mathrm{m})$ region as well as in the 12 and $25 \mu \mathrm{m}$ IRAS bands. It is concluded that the infrared evidence for interstellar PAHs and PAH-related species is compelling.
\end{abstract}

\section{INTRODUCTION}

The scientific organizing committee has allocated one entire day of this 5 day symposium to the interstellar emission bands at 3050, 1610, "1310," 1160 and $885 \mathrm{~cm}^{-1}$ (3.28, 6.2, "7.7," 8.6 and 11.3 microns) because the bands provide very detailed information about an apparently ubiquitous and abundant "dust" component. The bands are associated with a large number of different types of astronomical object (Bregman, 1989; Roche, 1989; and Sellgren, 1989; and references therein) implying that they are due to a surprisingly widespread, extremely stable, interstellar constituent. While it has been recognized for some time now that the emission was most likely due to IR fluorescence from molecule-sized species pumped by UV photons (Allamandola, Greenberg and Norman, 1979; Sellgren, 1984), the identity of the carriers has remained enigmatic. Current thinking that polycyclic aromatic hydrocarbons (PAHs) and related materials are the long sought after carriers can be traced to the suggestion of Duley and Williams (1981) that they arise from the vibrations of chemical subgroups attached to the aromatic moieties at the surfaces of small $(<0.01 \mu \mathrm{m})$, amorphous carbon particles. Subsequently Léger and Puget (1984) and Allamandola, Tielens and Barker (1985) (hereafter LP and ATB respectively) proposed that individual molecule-sized $(>0.001 \mu \mathrm{m})$ PAHs and PAH related materials were the carriers. The principal reason for this assignment was the resemblance of the interstellar emission spectra with the vibrational spectra of these materials. Related observations support an aromatic carrier as well. The fraction of total IR luminosity radiated in the " $1310^{\prime \prime} \mathrm{cm}^{-1}$ ( $" 7.7^{\prime \prime} \mu \mathrm{m}$ ) feature, which is by far the most intense of the bands, is strongly correlated with the carbon available 
TABLE 1: EMISSION COMPONENTS: PROPERTIES AND ASSIGNMENTS ${ }^{1}$

\begin{tabular}{cccc}
\hline \hline $\begin{array}{c}\lambda \\
\left(\mathrm{cm}^{-1}\right)\end{array}$ & $\begin{array}{c}\lambda \\
(\text { Microns })\end{array}$ & $\begin{array}{c}\text { FWHH } \\
\left(\mathrm{cm}^{-1}\right)\end{array}$ & ASSIGNMENT $^{2}$ \\
\hline
\end{tabular}

THE MAJOR BANDS

\begin{tabular}{|c|c|c|c|}
\hline 3040 & 3.29 & 30 & Aromatic $C-H$ stretch $(v=1 \rightarrow v=0)$ \\
\hline 1615 & 6.2 & 30 & Aromatic $C-C$ stretch \\
\hline $1315-1250$ & 7.6-8.0 & $70-200$ & Blending of several strong aromatic $C-C$ stretching bands \\
\hline 1150 & 8.7 & - & Aromatic $C-H$ in-plane bend \\
\hline 885 & 11.3 & 30 & Aromatic $C-H$ out-of-plane bend for non-adjacent, peripheral \\
\hline \multicolumn{4}{|c|}{ THE MINOR FEATURES } \\
\hline 3085 & 3.24 & - & $\begin{array}{l}\text { Overtone and/or combination involving fundamentals in } \\
\text { the } 1810-1050 \mathrm{~cm}^{-1}(5.52-9.52 \mu \mathrm{m}) \text { range }\end{array}$ \\
\hline 2995 & 3.34 & - & $\begin{array}{l}\text { Overtone and/or combination involving fundamentals in } \\
\text { the } 1810-1050 \mathrm{~cm}^{-1}(5.52-9.52 \mu \mathrm{m}) \text { range }\end{array}$ \\
\hline 2940 & 3.4 & “20" & Aromatic $C H$ stretch $(v=2 \rightarrow v=1)$ \\
\hline 2890 & 3.46 & - & $\begin{array}{l}\text { Overtone/combination band involving fundamentals in } \\
\text { the } 1810-1050 \mathrm{~cm}^{-1}(5.52-9.52 \mu \mathrm{m}) \text { range, } \\
\text { aromatic } C H \text { stretch (high } v \text { ), aliphatic } C H \text { stretch,? }\end{array}$ \\
\hline 2850 & 3.51 & - & $\begin{array}{l}\text { Aromatic } C H \text { stretch }(v=3 \rightarrow v=2) \text {, aliphatic } C H \text { stretch, } \\
\text { overtone/combination band involving fundamentals in } \\
\text { the } 1810-1050 \mathrm{~cm}^{-1}(5.52-9.52 \mu \mathrm{m}) \text { range }\end{array}$ \\
\hline 2810 & 3.56 & - & $\begin{array}{l}\text { Aromatic } C H \text { stretch (high } v \text { ), aldehydic } C H \text { stretch, } \\
\text { overtone/combination band involving fundamentals in } \\
\text { the } 1810-1050 \mathrm{~cm}^{-1}(5.52-9.52 \mu \mathrm{m}) \text { range }\end{array}$ \\
\hline $1960-1890$ & $5.1-5.3$ & 30 & Combination of $\mathrm{CH}$ out-of-plane and in-plane bend,? \\
\hline 1785-1755 & $5.6-5.7$ & 40 & $\begin{array}{l}\text { Overtone of } 885 \mathrm{~cm}^{-1}(11.3 \mu \mathrm{m}) \text { band; Aromatic } C-C \text { stretch; } \\
\text { Carbonyl } C=0 \text { stretch,? }\end{array}$ \\
\hline $1470-1450$ & 6.8-6.9 & 30 & Aromatic $C-C$ stretch, aliphatic $C H$ deformation \\
\hline 840 & 11.9 & - & $C-H$ out-of-plane bend for doubly adjacent $H$ atoms \\
\hline 790 & 12.7 & - & $C-H$ out-of-plane bend for triply adjacent $H$ atoms \\
\hline \multicolumn{4}{|c|}{ THE BROAD COMPONENTS } \\
\hline 2940 & 3.5 & & Overlap of $C-H$ stretching modes, shifted by anharmonic effects, \\
\hline $3115-2740^{\dagger}$ & $3.21-3.65^{\dagger}$ & “300" & $\begin{array}{l}\text { with overtones and combinations of } C-C \text { stretch fundamentals } \\
\text { in the } 1670-1250 \mathrm{~cm}^{-1}(6-8 \mu \mathrm{m}) \text { region, aliphatic } C H \text { stretch?,? }\end{array}$ \\
\hline$\sim 1200$ & $\sim 8.5$ & $“ 400 "$ & Blending of many weak aromatic $C-C$ stretching bands \\
\hline $1810-1050^{\dagger}$ & $5.52-9.52 \dagger$ & & \\
\hline 880 & 12 & $" 160 "$ & Overlap of many aromatic $C-H$ out-of-plane bending \\
\hline $950-740^{\dagger}$ & $10.5-13.5^{\dagger}$ & & $\begin{array}{l}\text { modes for non-adjacent as well as } \\
\text { doubly and triply adjacent peripheral } H \text {-atoms }\end{array}$ \\
\hline
\end{tabular}

Red-Near IR Continuum Electronic transitions between low-lying levels in ionized and complexed PAHs and amorphous carbon particles

Mid-IR Continuum

Quasi-continuum formed by overlapping overtone and combination bands

" "Value estimated from several published spectra.

$\dagger$ Rough limits of the feature.

1: When the assignment is not clear, several possible explanations are listed. The first seems most likely.

2: This table is extensively discussed in Allamandola, Tielens, and Barker, 1989. 
in planetary nebulae (Cohen et al., 1986). As the carriers must be produced under harsh conditions in planetary nebulae, they must be extremely stable. Furthermore, although there is some variation among the relative band intensities, the bands are correlated implying that a single class of chemical species is responsible (Cohen et al., 1986). These observations are completely consistent with PAHs which are a family of extremely stable, planar hydrocarbons which have a chicken wire-like structure.

Interstellar PAHs are thought to be very abundant, more so in fact than all of the known interstellar, gaseous, polyatomic molecules combined (LP; ATB). The proposed ubiquity and high abundance of interstellar PAHs implies that their influence on various processes and phenomena can be profound (Omont, 1986). For example they may be responsible for the infrared cirrus discovered by the IRAS satellite (ATB; Puget, Léger and Boulanger, 1985), some of the diffuse interstellar bands in the visible (van der Zwet and Allamandola, 1985; d'Hendecourt and Léger, 1985; Crawford, Tielens, and Allamandola, 1985), maintaining interstellar cloud temperatures (d'Hendecourt and Léger, 1987), moderating interstellar cloud chemistry (Lepp and Dalgarno, 1987), and contributing to the deuterium enrichments found in interplanetary dust particles and meteorites (Allamandola, Sandford and Wopenka, 1987; Allamandola, Tielens and Barker, 1989b).

In order to ascertain just how seriously one should take these suggestions it is important to consider the spectroscopic arguments in favor of PAHs. While definitive evidence is lacking in the ultraviolet and visible spectral regions (Donn, Allen, and Khanna, 1989; Léger et al., 1989), the case in the infrared is now compelling. Several predictions have been borne out and the theory continues to be capable of accommodating new observational results. Some of the recent additions to the evidence that PAHs and PAH-related species are responsible for many aspects of the infrared emission spectrum will be reviewed in this paper.

\section{INTERSTELLAR SPECTRA AND THE SPECTRA OF PAHS}

The interstellar emission spectra consist of strong, well-defined bands, weak bands, and broad features. To date, the relative intensities of the strong bands appear to be reasonably well-correlated. This is not the case for the weak bands and broad features. Any model which attempts to explain the IR emission phenomenon should address all of these characteristics. With one exception, the proposals skirt most of the issues of spectroscopic detail. There are several interpretations of the emission phenomenon based on PAHs. Duley and Williams (1988, 1989), while not specifically addressing the weak bands and broad features, envision that the well-defined bands arise from PAHs and side-groups on the PAHs which comprise hydrogenated amorphous carbon (HAC) particles. Léger and d'Hendecourt (1987, 1989), again principally addressing the well-defined bands, envision that they arise from free, molecule-sized PAHs containing between 50-100 carbon atoms and not PAHs which are part of carbonaceous particles. Allamandola, Tielens and Barker (1987, and this paper) envision that smaller, free, ionized PAHs, containing between 20-50 carbon atoms, are responsible for both the well defined and weak bands but larger PAHs, PAH clusters, and/or amorphous carbon particles, containing on the order of 400 carbon atoms, contribute to the broad features. Whether the PAHs are free or part of a larger particle is, at present, controversial. For the emission to originate from the aromatic structural units of a particle (HAC), or PAH cluster, 
requires that the vibrational energy created by the absorption of the exciting photon remain localized in a specific $\mathrm{PAH}$ unit long enough for it to radiatively relax in the IR $\left(\approx 10^{-2} \mathrm{~s}\right)$. The principle objection to this is that the timescale for normal nonradiative relaxation in a solid is usually many orders of magnitude faster. While one might imagine that some individual PAH units may be very loosely coupled to the remainder of the carbon particle, this must be demonstrated experimentally before it can be accepted. On the other hand, IR emission from highly vibrationally excited, free molecules is expected. In fact, the detection of UV-pumped IR emission from the bicyclic aromatic molecule azulene has been reported by Cherchneff and Barker (1989).

In view of the space limitation, this article will not deal with the differences between the various models but rather focus on the underlying, unifying theme that PAHs and PAH-like species are responsible for the emission. Furthermore, rather than discuss the general assignments yet again, this paper will focus on the spectroscopic issues raised by recent observational and experimental developments. All of the IR emission features and assignments are summarized in Table 1. The emission spectrum from position 4 in the Orion Bar is compared with the absorption spectra of several PAHs in Figure 1. Although the interstellar spectra resemble the laboratory data, they do not match in detail. Some questions raised by a careful comparison of the interstellar spectra with a random set of laboratory spectra of PAHs are:

1. Why does the " 1310 " $\mathrm{cm}^{-1}$ ( " $7.7 " \mu \mathrm{m}$ ) feature seem so poorly reproduced?

2. Why do so few of the bands in the $910-710 \mathrm{~cm}^{-1}(11-14 \mu \mathrm{m})$ range in the laboratory spectra match the single interstellar band at $885 \mathrm{~cm}^{-1}(11.3 \mu \mathrm{m})$ and why are the laboratory features far more intense with respect to the bands at higher frequencies than in the interstellar case?

3. All of the laboratory spectra show a weak to moderate band near $1900 \mathrm{~cm}^{-1}$ (5.2-5.3 $\mu \mathrm{m})$, is there a corresponding interstellar feature?

In addition, recent observations show specific details which are not immediately apparent from the laboratory data. The questions raised here are:

1. Can PAHs account for both the spectral and spatial variations found in the $3230-2750 \mathrm{~cm}^{-1}(3.1-3.6 \mu \mathrm{m})$ region?

2. Can PAHs account for the spatial variation in the IRAS 12 and $25 \mu \mathrm{m}$ bands in reflection nebulae and the IR cirrus?

Each of these will be discussed in turn. It will be shown that the properties of PAHs permit one to satisfactorily answer all of these questions.

\subsection{THE “1910" $\mathrm{cm}^{-1}$ (“7.7” $\left.\mu \mathrm{m}\right) B A N D$}

The principal difficulty here is to select a mixture of PAHs which can reproduce the position and profile of the interstellar feature. In the interstellar medium less stable PAHs will be weeded out, leaving a mixture dominated by the most stable structures. The most stable PAHs are symmetric and in the most condensed form 


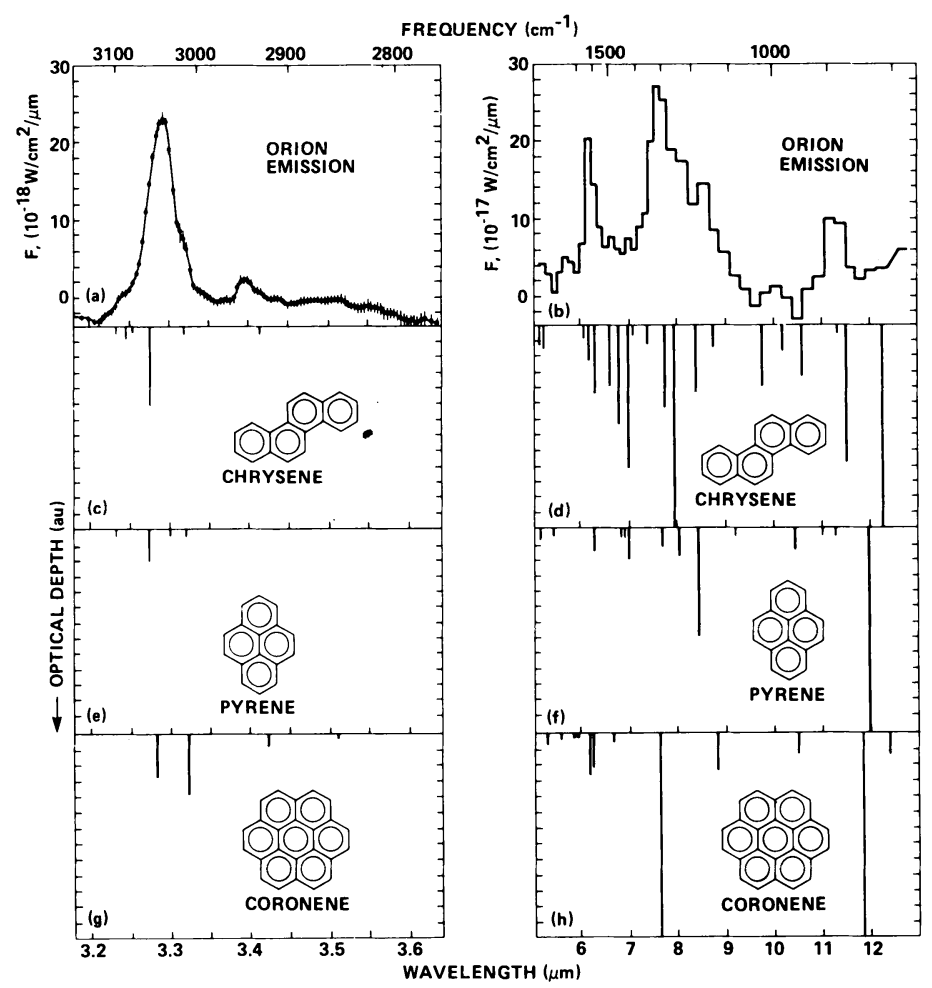

Fig. 1. The 3-13 micron emission spectrum from the Orion Bar compared with the absorption spectra of the PAHs chrysene, pyrene and coronene suspended in $\mathrm{KBr}$ pellets. (Orion, Bregman et al., 1988; Chrysene, Cyvin et al., 1982a; Pyrene, Cyvin et al., 1979; Coronene, Bakke et al., 1979; Cyvin et al., 1982b). Figure reproduced from Allamandola, Tielens and Barker, 1987.

(van der Zwet and Allamandola, 1985; Crawford, Tielens, and Allamandola, 1985). One example of a mixture comprised largely of the most stable molecular forms is the soot formed in the high temperature combustion of hydrocarbons. Figure 2 shows a comparison of the IR emission spectrum in the $1670-1250 \mathrm{~cm}^{-1}(6-8 \mu \mathrm{m})$ region from Orion with the Raman spectrum of auto soot.

The Raman spectrum of soot principally probes the aromatic carbon-carbon stretching vibrational frequencies because the Raman scattering cross section for these bonds is very large and they are the most dominant type of bond in the mixture. This comparison dramatically shows that the $C-C$ vibrational frequencies in a mixture of the more stable PAHs coincides quite well with the frequency range of the interstellar features (ATB). The confident assignment of this interstellar feature primarily to symmetric interstellar PAHs is supported by the good match of the major emission bands in the 2000 to $1000 \mathrm{~cm}^{-1}(5-10 \mu \mathrm{m})$ region from NGC 2023 (dots) with the calculated infrared emission spectra from 4 symmetric PAHs shown in Figure 3 (Léger and d'Hendecourt, 1987). 


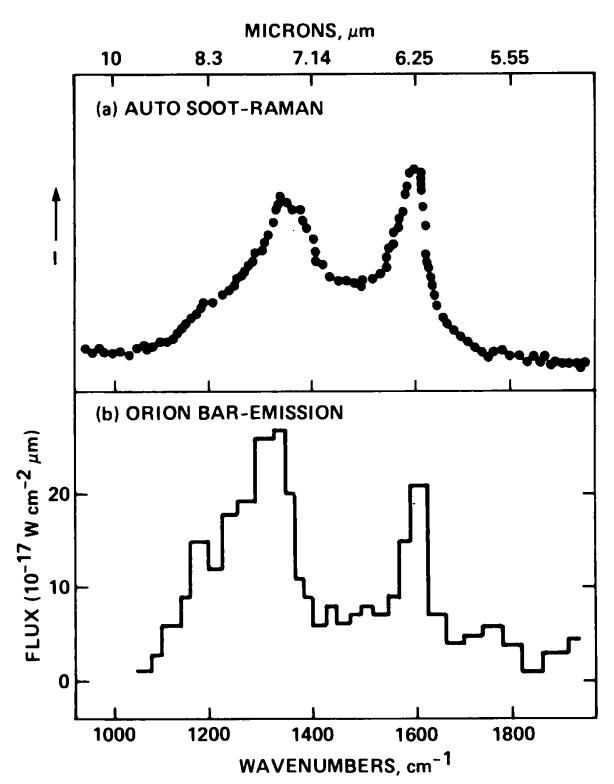

Fig. 2. Comparison of the 5 to 10 micron Raman spectrum of auto soot (a form of amorphous carbon which is rich in the more stable PAHs) with the emission from Orion (soot spectrum, adapted from Rosen and Novakov, 1978; Orion, Bregman et al., 1988). Figure reproduced from Allamandola, Tielens and Barker, 1985.

\subsection{THE $900-500 \mathrm{~cm}^{-1}(11-20 \mu \mathrm{m})$ REGION}

\subsubsection{The $900-500 \mathrm{~cm}^{-1}(11-20 \mu \mathrm{m})$ band frequencies}

The spectra of PAHs studied in the laboratory have several bands in this region because they have more than one $H$ atom per edge ring. These bands have long been used by chemists as a diagnostic of the number of adjacent hydrogen atoms on the edge rings of aromatic molecules. One difficulty in assigning the emission in this region to PAHs arose because most interstellar spectra appeared to have only one band in this region at a position $\left(885 \mathrm{~cm}^{-1}, 11.3 \mu \mathrm{m}\right)$ which implied one hydrogen per ring. This was taken to indicate severe dehydrogenation in spite of the fact that hydrogen is greater than $10^{7}$ times more abundant than the proposed PAHs.

This problem was resolved when it was shown that IRAS spectra of objects emitting the bands also have a broad plateau of emission from about $950-770 \mathrm{~cm}^{-1}$ $(10.5-13 \mu \mathrm{m})$ (Cohen, Tielens, and Allamandola, 1985). This relieved the difficulties associated with invoking partial dehydrogenation. This also implied that the interstellar PAHs responsible had edge rings with single, as well as doubly and triply adjacent $H$ atoms, but not four or five, placing strong constraints on the molecular structures possible and again indicating that the most stable, symmetric forms are dominant in the interstellar medium. 


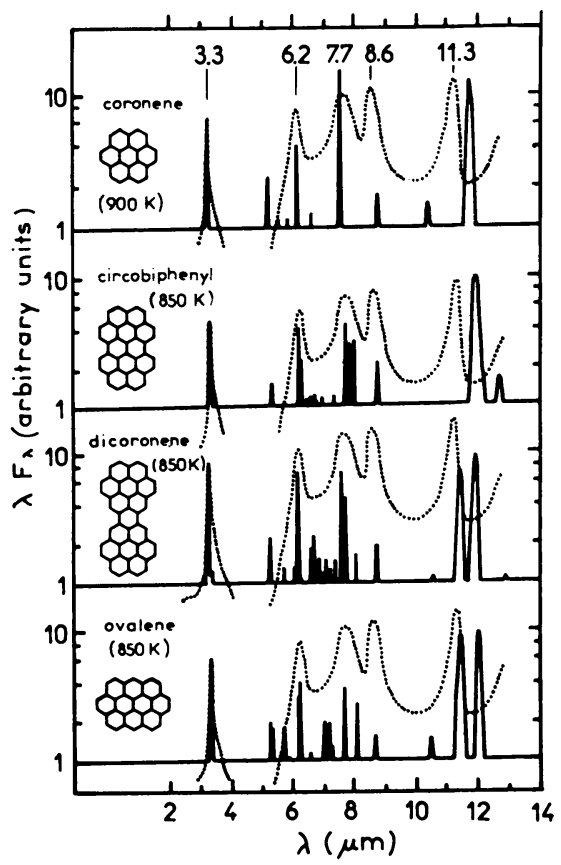

Fig. 8. Emission spectra of several compact PAHs calculated from laboratory absorption spectra measured at room temperature compared to the observed spectrum of the reflection nebula NGC 2023 (dots). Figure reproduced from Léger and d'Hendecourt, 1987.

Recent observations by Witteborn et al. (1989) and Roche, Aitken, and Smith (1989) have shown that there is resolvable structure on this plateau. The new features are just where one would expect to find them for PAHs with doubly and triply adjacent $H$ atoms per edge-ring: in the $840-830 \mathrm{~cm}^{-1}(11.9-12 \mu \mathrm{m})$ and $790-775 \mathrm{~cm}^{-1}(12.6-12.9 \mu \mathrm{m})$ regions.

\subsubsection{The $900-500 \mathrm{~cm}^{-1}(11-20 \mu \mathrm{m})$ band intensities}

The interstellar 950-770 $\mathrm{cm}^{-1}(10.5-13 \mu \mathrm{m})$ plateau and $885 \mathrm{~cm}^{-1}(11.3 \mu \mathrm{m})$ band are generally much less intense than the " $1310^{\prime \prime} \mathrm{cm}^{-1}(" 7.7 " \mu \mathrm{m})$ feature whereas the opposite is true in the laboratory spectra. This discrepancy has been shown to be the result of a spectral artifact produced by the techniques used to measure most of the PAH spectra published to date. Most laboratory spectra are obtained by mixing PAH crystallites with salt and pressing the mixture into a transparent pellet. Individual PAH molecules are not isolated in this way and molecular interactions between adjacent PAHs produce large band strength enhancements in the $900-500 \mathrm{~cm}^{-1}(11-20 \mu \mathrm{m})$ region. (These experiments, reported by Sandford and 
Allamandola at the Wyoming Summer School on Interstellar Processes held in July, 1986, are described in detail in Allamandola and Sandford, 1988).

This property is not only important because it reconciles an apparently serious inconsistency with the PAH hypothesis but also because it provides a powerful spectroscopic argument in favor of the well-defined bands arising from free, molecule-sized species rather than particles (in which interactions would be expected to enhance emission in this region).

\subsection{THE $1900 \mathrm{~cm}^{-1}(5.2-5.9 \mu \mathrm{m})$ FEATURE}

As most laboratory spectra of PAHs show a weak band close to this position, the PAH hypotheses predicts an interstellar band here. This was recently searched for, and found in the spectrum of $\mathrm{BD}+30^{\circ} 3639$, an object which emits the well-defined bands (see Bregman, 1989; and Allamandola et al., 1989b).

\subsection{THE SPECTRAL AND SPATIAL VARIATIONS IN THE $3230-2750 \mathrm{~cm}^{-1}$ (3.1-3.6 $\mu \mathrm{m})$ REGION}

In addition to the prominent $3050 \mathrm{~cm}^{-1}(3.28 \mu \mathrm{m})$ band and the broad, weak component from 3225-2780 $\mathrm{cm}^{-1}(3.1-3.6 \mu \mathrm{m})$, several weak features have been discovered in this region as well (Geballe et al., 1985; de Muizon et al., 1986). The weak features are superimposed on the broad component and can arise from several types of vibrations associated with PAHs. These include: combinations and overtones involving $C C$ stretching fundamentals in the $1700-1100 \mathrm{~cm}^{-1}(5.9-9 \mu \mathrm{m})$ range, $v=3 \rightarrow 2$ and $2 \rightarrow 1$ aromatic $C H$ stretch transitions in the most highly vibrationally excited PAHs, and $v=1 \rightarrow 0$ aliphatic $C H$ stretch transitions for aliphatic side-groups attached to PAHs (de Muizon et al., 1986; Barker, Allamandola, and Tielens, 1987).

The relative intensities of all of these features have recently been found to vary as a function of position near the Orion Bar and near the star exciting the Red Rectangle, indicating that several components are responsible (Geballe et al., 1989). These observations, which can be accommodated by the PAH hypothesis in a straightforward way, yield insight into the size and photochemical evolution (dehydrogenation) of interstellar PAHs. The spectral and spatial variations imply that overtones and combinations of $C C$ stretching bands and aromatic $C H v=3 \rightarrow 2$ and $2 \rightarrow 1$ transitions in PAHs with 20-50 carbon atoms contribute to the broad component and weaker features in both objects. Aliphatic $C H$ stretching vibrations in side groups can also contribute in the Orion Bar but are difficult to reconcile with the observed variations in the Red Rectangle (Geballe et al., 1989).

\subsection{THE SPATIAL VARIATION IN THE 12 AND $25 \mu$ m IRAS BAND INTEN- SITIES IN REFLECTION NEBULAE AND THE IR CIRRUS}

The intensity of the $12 \mu \mathrm{m}$ IRAS band drops with respect to that of the $25 \mu \mathrm{m}$ band in the vicinity of the exciting star in the case of reflection nebulae (Sellgren, 1989; and Luan et al., 1989) and close to hot stars in the case of the cirrus (Puget, 1989; and Boulanger et al., 1988). The relative intensities are also found to vary between 

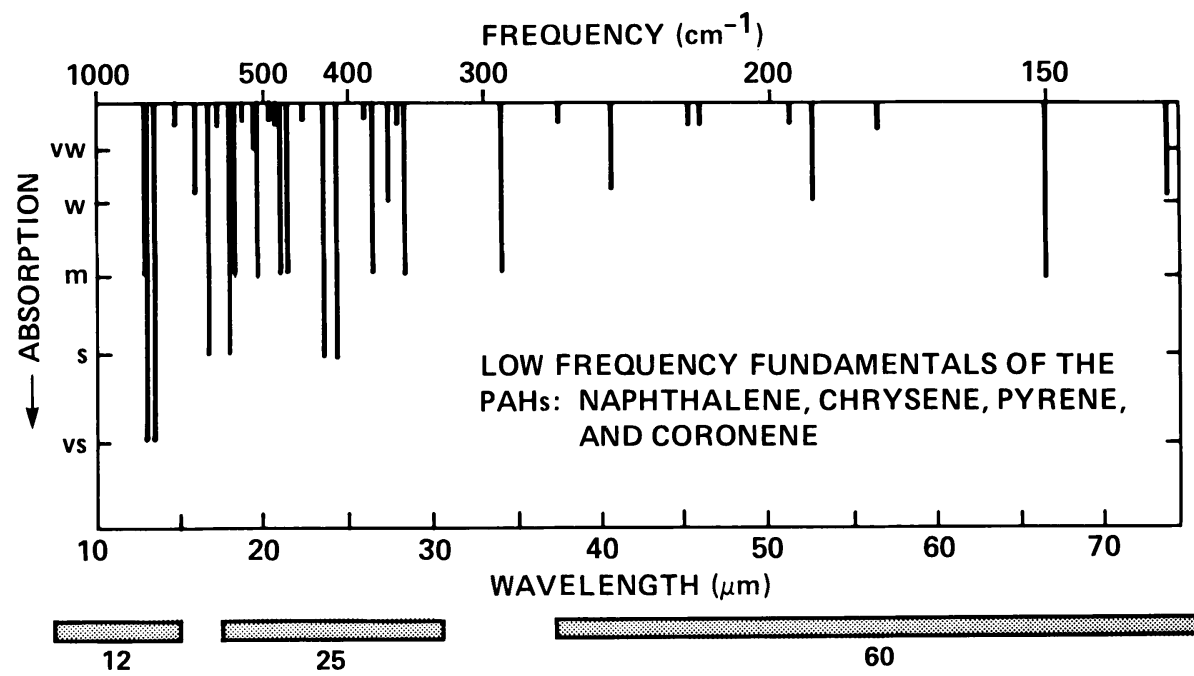

60

IRAS BANDS

C-H BENDING

C-C-C OUT-OF-PLANE BENDING

Fig. 4. A schematic composite absorption spectrum for the out-of-plane vibrations in the mixture of aromatic molecules naphthalene, chrysene, pyrene and coronene in the $1000-130 \mathrm{~cm}^{-1}(10-75 \mu \mathrm{m})$ range compared to the IRAS bands. The regions in which the $C H$ in- and out-of-plane and carbon plane bending vibrations fall are also indicated. Figure adapted from Allamandola, Tielens and Barker, 1987.

clouds of IR "cirrus" (Verter, Magnagni, and Dwek, 1989). If PAHs and PAHrelated materials are responsible for the emission, this behavior can be understood in terms of dehydrogenation rather than grain destruction. As shown in Figure 4, the $12 \mu \mathrm{m}$ IRAS band covers the $1250-670 \mathrm{~cm}^{-1}(8-15 \mu \mathrm{m})$ range which primarily encompasses the $\mathrm{CH}$ in-plane and out-of-plane bending vibrations in PAHs while the $25 \mu \mathrm{m}$ IRAS band covers the $560-330 \mathrm{~cm}^{-1}(18-30 \mu \mathrm{m})$ region which overlaps with many of the $C-C-C$ aromatic, plane bending vibrations of PAHs (Allamandola, Tielens and Barker, 1987). Consequently, if a PAH is stripped of hydrogen it will lose most of its IR activity in the $1000-500 \mathrm{~cm}^{-1}(10-20 \mu \mathrm{m})$ region (IRAS $12 \mu \mathrm{m}$ band) but not in the $500-10 \mathrm{~cm}^{-1}(20-1000 \mu \mathrm{m})$ region. Small PAHs (15-30 carbon atoms) contribute strongly to the infrared emission because they are the most highly vibrationally excited by the absorption of an ultraviolet photon. These are also the PAHs which will be the first to suffer $H$ atom loss if sufficiently excited (Tielens et al., 1987; Allamandola, Tielens, and Barker, 1989b). Consequently, if PAHs are indeed responsible for this emission, the drop in intensity of the $12 \mu \mathrm{m}$ IRAS band with respect to the $25 \mu \mathrm{m}$ IRAS band can be understood in terms of the dehydrogenation of the most intensely emitting PAHs and not the destruction of the carbon skeleton or of a particular class of grains. By inverting the dehydrogenation treatment described in Geballe et al. (1989), one can probe the strength 
of the interstellar radiation fields in these regions.

This explanation can be tested, provided the spatial behavior of the $3050 \mathrm{~cm}^{-1}$ $(3.28 \mu \mathrm{m})$ band can be measured. As this band is due to the aromatic $C H$ stretch, it too should show a similar drop in intensity with respect to the predominantly $C C$ modes in the $1670-1180 \mathrm{~cm}^{-1}(6-8.5 \mu \mathrm{m})$ and $500-10 \mathrm{~cm}^{-1}(20-1000 \mu \mathrm{m})$ regions.

\section{CONCLUSIONS}

Most of the spectroscopic objections to PAHs carrying the infrared emission bands can be satisfactorily answered. The general arguments in favor of PAHs and PAHrelated materials being responsible for most, if not all, of the spectral features are compelling (Table 3). Nonetheless some important questions have recently been raised. For example, what is the reason that the width of the $3050 \mathrm{~cm}^{-1}$ $(3.28 \mu \mathrm{m})$ band may vary significantly (Tokunaga et al., 1988), do QCC's contain PAHs (Sakata, 1989), do QCC's and amorphous carbon particles show the fine spectroscopic details and variations that free PAHs do (Bussoletti and Colangeli, 1989) and are the PAH structural units in amorphous carbon particles indeed loosely enough bound to permit them to radiate as free molecules (Duley, 1989)? As with most of the previous questions (which were originally perceived to be condemning to the PAH hypothesis), the answers to these should provide further insight into the nature of interstellar PAHs and the environments from which they emit.

ACKNOWLEDGEMENTS. This work has been supported by NASA Grant 188415700.

\section{REFERENCES}

Allamandola, L. J., Bregman, J., Sandford, S. A., Tielens, A. G. G. M., Witteborn, F. C., Wooden, D. H., and Rank, D. $1989 a$, Ap. J., submitted.

Allamandola, L., Tielens, A. G. G. M. and Barker, J. R. 1989b, Ap. J. Suppl., in press.

- 1987 in Physical Processes in Interstellar Clouds, eds. Morfill, G. E. and Scholer, M. (Dordrecht: Reidel), p. 305.

1985, Ap. J., 290, L25.

Allamandola, L. J., and Sandford, S. A. 1988, in Dust in the Universe, eds. Bailey, M. A. and Williams, D. A. (Cambridge: Cambridge University Press), in press.

Allamandola, L. J., Sandford, S. A., and Wopenka, B. 1987, Science, 237, 56.

Allamandola, L. J., Greenberg, J. M. and Norman, C. A. 1979, Astr. Ap., 7, 66.

Bakke, A., Cyvin, B. N., Whitmer, J. C., Cyvin, S. J., Gustavsen, J. E. and Klaeboe, P. $1979, Z$. Naturforsch, 84a, 579.

Barker, J. R., Allamandola, L. J. and Tielens, A. G. G. M. 1987, Ap. J. (Letters), 815, L61.

Boulanger, F., Beichman, C., Désert, F. X., Helou, G., Perault, M. and Ryter, C. 1988, Ap. J., submitted.

Bregman, J. D. 1989, in IAU Symposium 195: Interstellar Dust, eds. Allamandola, L. J. and Tielens, A. G. G. M., (Dordrecht: Kluwer), p. 109.

Bregman, J. D., Allamandola, L. J., Tielens, A. G. G. M., Rank, D. M. and Geballe, T. R. 1988, Ap. $J .$, in press.

Bussoletti, E. and Colangeli, L. 1989, in IAU Symposium 195: Interstellar Dust, eds. Allamandola, L. J. and Tielens, A. G. G. M., (Dordrecht: Kluwer), p. 147.

Cherchneff, I. and Barker, J. R. 1989, in Interstellar Dust Contributed Papers, eds. A. G. G. M. Tielens and L. J. Allamandola, NASA CP-3036.

Cohen, M., Tielens, A. G. G. M., Bregman, J. D., Witteborn, F. C., Rank, D. M., Allamandola, L. J., Wooden, D. and de Muizon, M. 1988, Ap. J., in press. 
Cohen, M., Allamandola, L. J., Tielens, A. G. G. M., Bregman, J. D., Simpson, J. P., Witteborn, F. C., Wooden, D. and Rank, D. 1986, Ap. J., 302, 737.

Cohen, M., Tielens, A. G. G. M., and Allamandola, L. J. 1985, Ap. J. (Letters), 299, L93.

Crawford, M. K., Tielens, A. G. G. M. and Allamandola, L. J. 1985, Ap. J. (Letters), 298, L45.

Cyvin, B. N., Klaeboe, P., Whitmer, J. C. and Cyvin, S. J. $1982 a, Z$. Naturforsch, 87a, 251.

Cyvin, S. J., Cyvin, B. N., Brunvoll, J., Whitmer, J. C., and Klaeboe, P. 1982b, Z. Naturforsch, $\mathbf{8 7 a}, 1359$.

Cyvin, S. J., Cyvin, B. N., Brunvoll, J., Whitmer, J. C., Klaeboe, P., and Gustavsen, J. E. 1979, Z. Naturforsch, 34a, 876.

de Muizon, M., Geballe, T. R., d'Hendecourt, L. B. and Baas, F. 1986, Ap. J. (Letters), 306, L105.

d'Hendecourt, L. B. and Léger, A. 1987, Astr. Ap., 180, L9.

Donn, B. D. Allen, Jr., J. E., and Khanna, R. K. 1989, in IAU Symposium 195: Interstellar Dust, eds. Allamandola, L. J. and Tielens, A. G. G. M., (Dordrecht: Kluwer), p. 181.

Duley, W. W. and Williams, D. A 1989, in IAU Symposium 195: Interstellar Dust, eds. Allamandola, L. J. and Tielens, A. G. G. M., (Dordrecht: Kluwer), p. 141. 1988, M. N. R. A. S., 196. 269.

Geballe, T. R., Tielens, A. G. G. M., Allamandola, L. J., Morehouse, A. and Brand, P. W. J. L. $1989, A p . J .$, in press.

Geballe, T. R., Lacy, J. H., Persson, S. E., McGregor, P. J. and Soifer, B. T. 1985, Ap. J., 292, 500 .

Gillett, F. C., Forrest, W. J., and Merrill, K. M. 1973, Ap. J., 188, 87,

Léger, A., Verstraete, L., d'Hendecourt, L., Défourneau, D., Dutuit, O., Schmidt, W., Lauer, J. C. 1989, in IAU Symposium 195: Interstellar Dust, eds. Allamandola, L. J. and Tielens, A. G. G. M., (Dordrecht: Kluwer), p.173.

Léger, A. and d'Hendecourt, L. B. 1987, in Polycyclic Aromatic Hydrocarbons and Astrophysics, eds. Léger, A., d'Hendecourt, L. B. and Boccara, N. (Dordrecht: Reidel), p. 223. 1985, Astr. Ap., 146, 81.

Léger, A. and Puget, J. L. 1984, Astr. Ap., 137, L5.

Lepp, S. and Dalgarno, A. 1988, Ap. J., \$24, 553.

Luan, L., Werner, M. W., Dwek, E., and Sellgren, K. 1989, in Interstellar Dust Contributed Papers, eds. A. G. G. M. Tielens and L. J. Allamandola, NASA CP-3036.

Omont, A. 1986, Astr. Ap., 164, 159.

Puget, J. L. 1989, in IAU Symposium 195: Interstellar Dust, eds. Allamandola, L. J. and Tielens, A. G. G. M., (Dordrecht: Kluwer), p. 119.

Puget, J. L., Léger, A. and Boulanger, F. 1985, Astr. Ap., 142, L19.

Roche, P. F. 1989, in IAU Symposium 195: Interstellar Dust, eds. Allamandola, L. J. and Tielens, A. G. G. M., (Dordrecht: Kluwer), p. 303.

Roche, P. F., Aitken, D. K. and Smith, C. H. 1989, M. N. R. A. S., in press.

Rosen, H. and Novakov, T. 1978, Atmos. Environment, 12, 923.

Sakata, A. and Wada, S. 1989, in IAU Symposium 185: Interstellar Dust, eds. Allamandola, L. J. and Tielens, A. G. G. M., (Dordrecht: Kluwer), p. 191.

Sellgren, K. 1989, in IAU Symposium 195: Interstellar Dust, eds. Allamandola, L. J. and Tielens, A. G. G. M., (Dordrecht: Kluwer), p. 103.

Sellgren, K. 1984, Ap. J., 277, 623.

Tielens, A. G. G. M., Allamandola, L. J., Barker, J. R. and Cohen, M. 1987, in Polycyclic Aromatic Hydrocarbons and Astrophysics, eds. Léger, A., d'Hendecourt, L. B., and Boccara, N. (Dordrecht: Reidel), p. 273.

Tokanaga, A. T., Nagata, T., Sellgren, K., Smith, R. G., Onaka, T., Nakada, Y., Sakata, A. and Wada, S. 1988, Ap. J., 828, 709 .

van der Zwet, G. P. and Allamandola, L. J. 1985, Astr. Ap., 146, 76.

Verter, F., Magnani, L. and Dwek, E. 1989, private communication.

Witteborn, F. C., Sandford, S. A., Bregman, J. D., Allamandola, L. J., Wooden, D. H., Cohen, M., Graps, A. L. 1989, Ap. J. in press. 


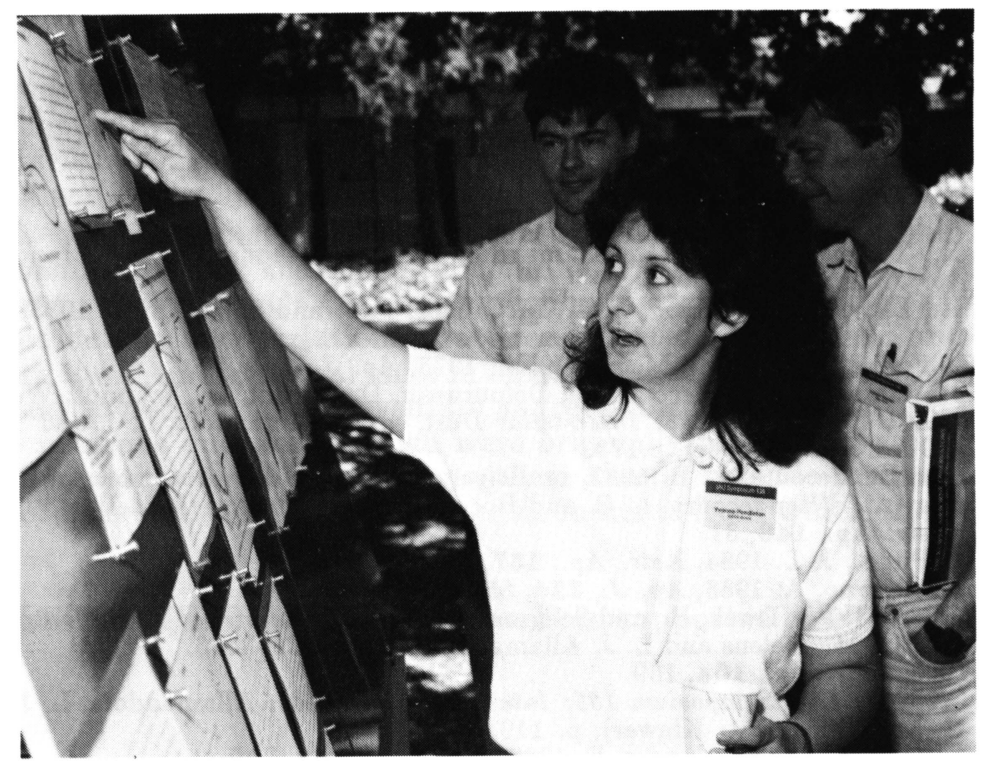

Yvonne Pendleton 